\title{
Integration study of high quality teaching resources in universities
}

\author{
Honglu Liu ${ }^{1}$, Shugang Zhang ${ }^{1}$, Xiaolan Guan ${ }^{2}$ \\ ${ }^{1}$ Beijing Jiaotong University, ${ }^{2}$ Beijing Institute of Graphic Communication (China) \\ blliu@,bjtu.edu.cn,.gjc.zsg@,bjedu.gov.cn, 08113101@,bjtu.edu.cn
}

Received: October 2011

Accepted: August 2012

\section{Abstract:}

Purpose: The development level and quality of education depend on the merits and efficiency in the use of teaching resources, especially in the case of obvious contradiction between the demand and supply of teaching resources. So to integrate teaching resources, improve the efficiency in the use of high quality teaching resources, and take the road of content development to enhance the competitiveness of education has become very important and urgent.

Design/methodology/approach: On the basis of analysis on the teaching resources of universities and the problems they faced, this paper introduced the basic concepts of cloud storage, and built the integration architecture of high quality teaching resources in universities based on the cloud storage.

Findings and Originality/value: The HDFS-based cloud storage proposed in this paper is a dynamically adjustable and Internet-based storage solution, and the users can access storage targets using the network through a common and easy-to-use protocol and application programming interfaces. This new technology is useful for end users benefits. With the continuous development and improvement of cloud storage, it will necessarily result in more and more applications in the institutions of higher learning and education network.

Originality/value: This paper introduced the cloud storage into the integration of high quality teaching resources in universities first and as a new form of service, it can be a good solution. 
Keywords: High quality teaching resources, Integration, Cloud storage, Information platform

\section{Introduction}

The teaching resources is not simply an economic concept, it is also a concept of education. The teaching resources refers to the sum of human, material and financial resources that can draw up occupied, used and consumed in the teaching activities. Resources is always the driving force for the resource owners to take activities, and due to the differences in the purpose and the ability to work for different resource owners, there are differences in the rationality of allocation and the efficiency of use. Therefore, the integration of resources that they have, especially high quality resources, is a strategic issue to any owner in the activities that must be considered.

There are some scholars began to introduce the optimal allocation theory into the field of educational research during the 1990s and used the integration approach in economics and management science to solve education problems. It has great theoretical and practical values, especially to the problem of reasonable utilization and allocation of teaching resources. The integration and optimal allocation of teaching resources is getting the attention of scholars.

But there is still not much literature about the teaching resources in universities, and most of the studies are still stuck at the macro level, lack of specific guidance to practical operability (Gao \& Wang, 2010). For example, literature "The Integration and Optimization of Teaching Resources in Beijing University of Chemical Technology" (Jia, 2008) and "Research on the Integration of College Teaching Resources" (Wang, 2009) put forward some strategies about how to promote the integration and optimization of China's university teaching resource using the theory of resource allocation and no boundary organization, and literature "Researching for Combination of the Resource of Science and Technology of Liaoning's College" (Ji, 2003) put forward four principles in the integration research of university resources.

In summary, even though the scholars both at home and abroad have got some achievements in the research about the integration and optimization of teaching resources, they are still not yet fully understand the principle of integration and optimal allocation, and there is still not a practical system about the strategy and method in the teaching resources integration and optimization. So this paper intends to take the high quality teaching resources in universities as a basic object of study. And on the basis of analysis on the teaching resources in universities and the problems they faced, it built the structural model for the integration information platform of high quality teaching resources in universities based on the theories and methods of cloud storage, and analyzed its operation mechanism. It aims to open up a new route for the integration of high quality teaching resources for the universities. 


\section{Theoretical Studies}

\subsection{The Concept of High Quality Teaching Resources in Universities}

Teaching Resources is the scientific use of economic concepts in the field of education. It refers to all the positive factors for educational purposes that can draw up in the educational activities. The meaning of teaching resources can be defined on the basis of resources, and from the educational requirement in the configuration of total community resources. However, many experts and scholars have put forward their views from different angles. In the book of "Simple Tutorial on the Economics of Education", it thought that the full meaning of teaching resources should include all the human resources, material resources and financial resources in the education that got during the social resource allocation. And this definition referred only to the currency embody of various resources. In the preface of the book "Human Resources Management in Education", the translator stressed that human, finance and material are the three resources of education in the traditional sense and with the progress of times, the information began to break into our lives in a more efficient posture and became a kind of invisible resources. It highlighted that the information is also an important resource for education on the basis of the other three resources.

According to the definitions and perspectives on teaching resources, the concept of teaching resources used in this paper refers specifically to the guarantee conditions of improving the quality and promoting the development of education, including the physical material resources, intangible ideas and ideology, shown in Figure 1.

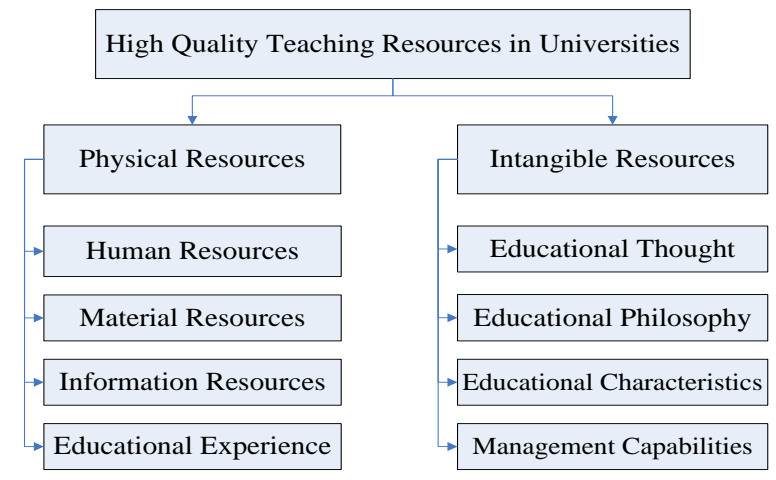

Figure 1 . The classification of high quality teaching resources in universities

\subsection{The Integration of High Quality Teaching Resources in Universities}

The teaching resources are the foundation of teaching work. With the continuous construction of teaching resources, some disadvantages are also emerging. Since the teaching resources has the features of large number and different forms, so the original organization mode of centralized management and storage can not adapt to the variety of practical needs of network development and resources applications, and it also results in the enormous resource waste due to the redundant construction of teaching resources. Therefore, there is an urgent need to integrate high quality teaching resources in the universities (Seppänen \& Mäkinen, 2012). By carrying out 
the integration of teaching resources, it can not only respond to the contradictions formed by the continuous growth of the teaching resource requirements and the relative shortage of the education investment and teaching resources due to the universities enrollment growth, but also can realize the sharing of teaching resources and improve the utilization efficiency of high quality teaching resources, so that there will be more access to quality educational opportunities for students.

The integration of teaching resources in universities is a new field of educational research, and it refers to the system engineering of improving the overall effectiveness through the adjustment, coordination and reorganization of existing teaching resources by optimizing allocation, and makes the main bodies with common mission and independent economic interests into a system besides the functions that related to each other but separated within the system in order to achieve coordinated development of size, structure, quality and effectiveness, promote the operation quality and overall level of education, enhance the school's strength and competitive advantage on the sharing of resources and interdisciplinary integration platform. The establishment of this development platform needs to carry out a full range of reconstruction and integration of teaching resources, such as the culture, curriculum, profession, organizational structure, human resources, and hardware facilities and so on, in order to form a long-term mechanism for sustainable development.

Meanwhile, the integration of high quality teaching resources in universities is by no means what a university can do, it particularly needs the local government departments attach great importance and guide actively, and also needs all the common efforts of the universities, the enterprises and the industry associations to expand the space for development through the innovation of the higher education system design, and strives to build the efficient open higher education body to meet the needs of economic development.

\section{Analysis of the Integration Information Platform of High Quality Teaching Resources Based on the Cloud Storage in Universities}

As a new form of service, the cloud storage can be a good solution to the integration of high quality teaching resources in universities. Using the cloud storage in the integration of high quality teaching resources in universities, it can not only save investment costs, simplify the setup and management tasks, but also can facilitate access from more places in order to achieve the teaching resource sharing.

\subsection{The Basic Concept of Cloud Storage}

Cloud storage is a new concept that extends and develops from the concept of cloud computing (Liu, Yu \& Zhu, 2011). The cloud storage takes the servers, storage devices and other hardware as a pool of resources by virtualization technology, rather than a discrete system, so that these resources can be assigned as needed. In this way, a storage cloud is equivalent to a virtual 
storage resource pool to accommodate different operating modes, and these modes can be quickly deployed to the physical facilities and dynamically call resources in accordance with the user needs. Cloud storage is a kind of storage that automatically splits the enormous amount of data into numerous smaller chunks of data and gives them to computer cluster with multiple storage resources for distributed storage. The core of cloud storage is a combination of application software, and achieves the transformation from the storage device to the storage service by the application software.

Using the cloud storage, the users don't have to know the model, interface, transmission protocols of the storage device or the number of disks in the storage system, namely, its type, capacity, storage devices and what kind of connection cable between servers like when they use a separate storage device. And the users don't have to establish the data backup system and disaster recovery system to ensure the data security and business continuity. In addition, the users also don't have to care about the state monitoring, maintenance, hardware and software updates and upgrades of the storage device. All devices in the cloud storage system are completely transparent to the users, and any authorized user can be connected to the cloud storage through a cable and access the data anywhere, shown in Figure 2.

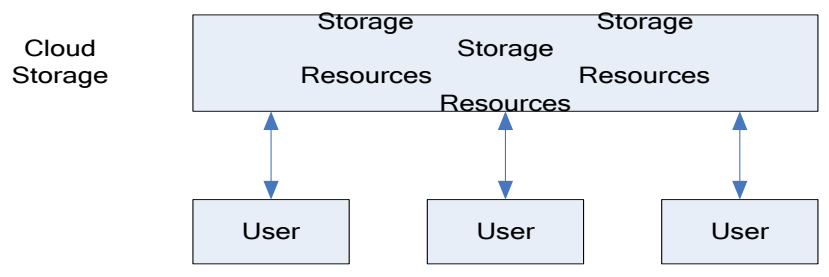

Figure 2. The cloud storage service

\subsection{The HDFS Architecture}

Among the numerous products and technologies of the cloud storage, the technology of HDFS (Hadoop Distributed the File System) can be implemented to run on ordinary PC clusters and effectively reduces the storage costs. This technology is the open source realization of Google File System (GFS), and it is the underlying implementation of distributed computing of the open-source framework Hadoop with high fault tolerance features (Chen, 2012). The HDFS can provide high throughput of data read and write, and thus it is suitable for those applications with large data sets. Therefore, the integration information platform for the universities constructed in this paper will also use this technology, shown in Figure 3.

HDFS takes the strategy of copies, and its purpose is to improve the system reliability and availability. There are three default copies in HDFS, one on the main node, one on another node in the same rack, and the other one on one node in a different rack. 


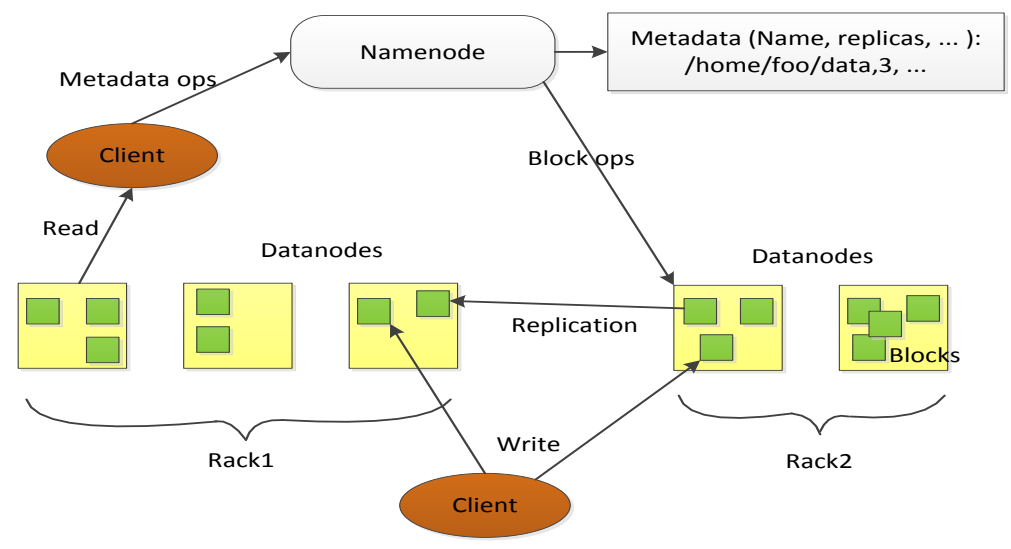

Figure 3. The HDFS architecture

\subsection{The Cloud Environment}

Before using the cloud storage to integrate the high quality teaching resources in universities, we have to build a cloud environment first. In this cloud environment, it provides the services of teaching resources store and access through the storage resources with dynamic scalability and virtualization (Wu, Fu, Ping \& Xie, 2011). Through the cloud, an enormous amount of teaching resources can be automatically split into smaller blocks of data and given to a large computer cluster system with multiple nodes for distributed storage.

The structural model of the integration and optimizing allocation platform of high quality teaching resources in universities based on the cloud storage can be divided into four layers, that is the storage layer, the fundamental management layer, the application interface layer and the access layer bottom-up respectively, shown in Figure 4 (Bian, Gao \& Shao, 2011).

\begin{tabular}{|c|c|c|c|}
\hline $\begin{array}{l}\text { The access } \\
\text { layer }\end{array}$ & $\begin{array}{l}\text { Personal Space } \\
\text { Services, Operator } \\
\text { Space Rental }\end{array}$ & $\begin{array}{c}\text { Data Backup, Data } \\
\text { Archiving, Centralized } \\
\text { Storage and Remote } \\
\text { Sharing of Enterprises } \\
\text { or SMB }\end{array}$ & $\begin{array}{l}\text { Video Surveillance, } \\
\text { Centralized Storage } \\
\text { Systems (such as } \\
\text { IPTV), High-capacity } \\
\text { Online Storage }\end{array}$ \\
\hline $\begin{array}{l}\text { The } \\
\text { application }\end{array}$ & \multicolumn{3}{|c|}{ Grid Access, User Authentication, Rights Management } \\
\hline layer & \multicolumn{3}{|c|}{ Public API Interface, Application Software, Web Service } \\
\hline $\begin{array}{c}\text { The } \\
\text { fundamental } \\
\text { management } \\
\text { layer }\end{array}$ & $\begin{array}{l}\text { Cluster System } \\
\text { Distributed File } \\
\text { System } \\
\text { Grid Computing }\end{array}$ & $\begin{array}{l}\text { Content Distribution } \\
\text { Data De-duplication } \\
\text { Data Compression }\end{array}$ & $\begin{array}{c}\text { Data Encryption } \\
\text { Data Backup } \\
\text { Data Disaster } \\
\text { Recovery }\end{array}$ \\
\hline \multirow{2}{*}{$\begin{array}{l}\text { The storage } \\
\text { layer }\end{array}$} & \multicolumn{3}{|c|}{$\begin{array}{l}\text { Condition Monitoring of Storage Virtualization, Centralized } \\
\text { Storage Management, Maintenance and Upgrade }\end{array}$} \\
\hline & \multicolumn{3}{|c|}{ The storage devices } \\
\hline
\end{tabular}

Figure 4. The structural model of the integration information platform of high quality teaching resources in universities based on the cloud storage

- The storage layer. The storage layer is the most fundamental part of the cloud storage. The storage devices can be fiber channel storage devices, or a NAS, iSCSI and other IP 
storage devices, and also can be a SCSI or SAS, DAS storage devices. The storage devices in the cloud storage are often numerous and distribute in different geographical locations linked with each other through the WAN, Internet or Fiber Channel network. There is a unified storage device management system above the storage devices that can realize the logic virtualization management, multi-link redundancy management, hardware status monitoring and troubleshooting of the storage device.

- The fundamental management layer. The fundamental management layer is the most central part of the cloud storage, and also the most difficult part to achieve. The fundamental management layer realizes the interoperability between the multiple storage devices in the cloud storage through clusters, distributed file system and grid computing technology in order to make multiple storage devices provide the same kind of external services, and also provide bigger, more powerful and better data access performance (Fu, Chen \& Wang, 2012). The content distribution systems and data encryption technology can ensure that the data in the cloud storage would not be accessed by unauthorized users, guarantee that the data would not lost by using data backup, disaster recovery technology and other measures, and thus ensure the security and stability of the cloud storage itself.

- The application interface layer. The application interface layer is the most flexible part of the cloud storage. Different operating units of cloud storage can develop different application service interfaces and offer a variety of application services based on the actual type of business, such as the video surveillance application platform, the video-on-demand application platform, the network drive reference platform, the remote data backup application platform and so on.

- The access layer. Any authorized user can login in to the cloud storage system through the standard common application interface, and enjoy the cloud storage service. And due to the difference of the operating units of the cloud storage, the access types and access methods are also different.

\section{Design and Development of the Integration Information Platform of High Quality Teaching Resources Based on the Cloud Storage in Universities}

\subsection{Security Architecture Design}

The security is a very important problem to be considered in the integration information platform of high quality teaching resources based on cloud storage in universities. There have to be appropriate protective measures between layers from the data transmission to storage. So in accordance with the cloud environment hierarchy of the integration information platform of high quality teaching resources in universities, we have to establish different protection strategies to protect the data that need to be stored, in order to achieve comprehensive protection from data 
transmission to the storage location (Lin \& Tzeng, 2012). The security architecture of the integration information platform of high quality teaching resources based on the cloud storage in universities uses the Information Dispersal Algorithms (IDA), distributed storage management, data bootstrap recovery and other technologies to realize the security storage management and transmission of data in different layers in the cloud storage, and its overall system security framework is shown in Figure 5.

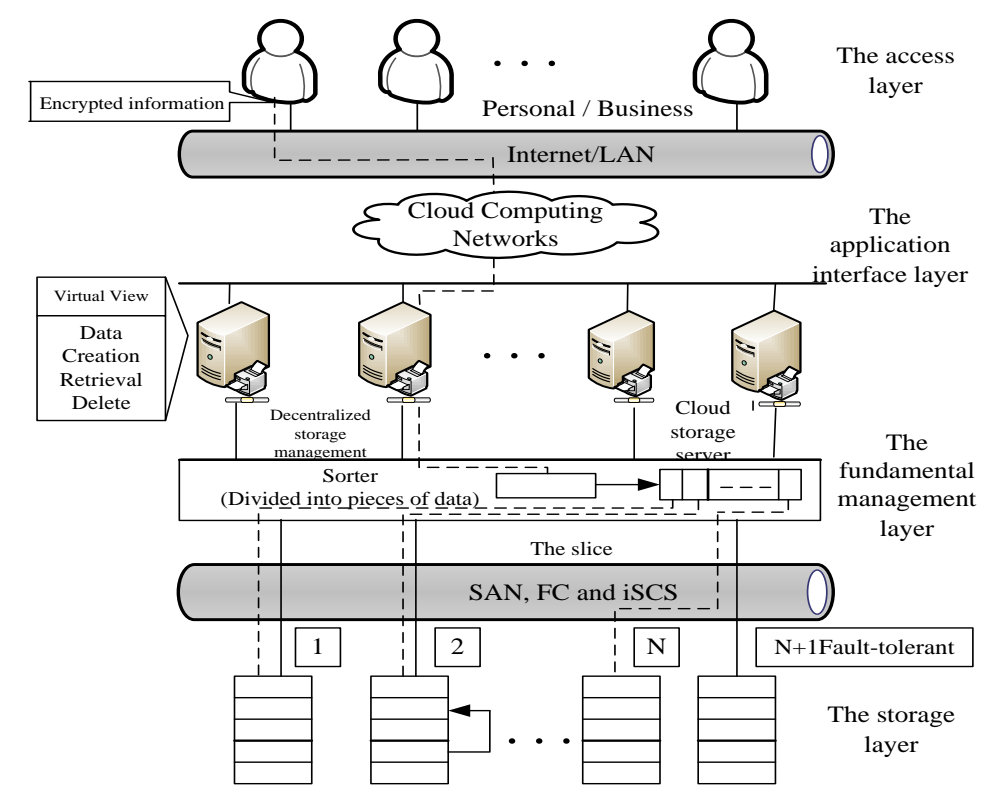

Figure 5. The security architecture of the integration information platform

\section{Design of the access layer to the application interface layer}

It can make the data got more security protection during the transmission in the network by access control and authentication using the encryption technology SSL to protect the storage data of the users. There are mutual authentication between the users and the cloud storage server. It identifies the safety certificate and identification of both sides, and then the user agent can use the data storage services after successful authentication through the security APIs and cloud communication connection.

\section{Design of the fundamental management layer}

Using the Information Dispersal Algorithms (IDA), the data slice will have relative confidentiality and security during transmission in the network and data storage. The fundamental management layer in the cloud storage fragments the stored information slice by slice using the sorter on the basis of IDA, and thus changes the data into data fragments that can not be identified by the other non-authentication systems. For each individual data fragments, these data fragments don't have any meaning, even if they are intercepted by others or accidentally stolen by implanted Trojan virus via scanning or on the storage device, since the interception party only gets part of the data fragments and the interception of the information does not have any real meaning, so that it can guarantee that there will not be leakage or spread of confidential 
information after data slice. In addition, when these data slices are put into memories in different geographical locations, even if other users extract by mistake, it still can protect the information not be analyzed. The distributed storage mechanism of data also makes the storage system have a certain degree of fault tolerance, disaster recovery capabilities, and thus improves the availability of information.

After the sorter fragments the data into slices according to the IDA, the cloud storage server will identify each data slice with a fixed handle of 64 byte, and these handles are unique. When reading data, it will create a data list in the master server according to the handle and byte range in the virtual view of the storage server to store the metadata in the system, including the information of file name that the users store, corresponding handle number and file size. The data storage are dispersed both in the content and in the storage devices, and when users need to access data in the cloud storage or operate, the Distributed Storage Manager (DSM) needs to integrate the distributed data and give users a virtual view. These dispersed data is transparent to users. The users can manage the data according to the view provided. In addition, DSM supports metadata management, and it facilitates the users to operate creation, retrieval and delete on the data.

\section{Design of the storage layer}

In order to achieve the security policy for data storage, it has to meet the needs of the users to store vast amounts of data in the design of the storage layer. And due to the growing in size and storage capacity of the storage system, the storage-related error rate will be higher and higher. So in order to ensure the high availability and reliability of cloud storage security system in data storage, the devices in the storage layer must be off-site storage and redundant, then it can improve the fault tolerance and storage utilization of the devices (Lu \& Shao, 2012). The system uses the Read-Solomon code to provide high error recovery techniques and ensure that the problem can be quickly detected after occurs. If the data on the device is damaged or lost, the storage systems will detect this problem automatically, and recalculate all the data by checking the piece available and then restore the corrupted data according to the integrity of the data in the other storage devices. It increases the mean time interval between failures of the cloud storage system through the bootstrap data recovery.

\subsection{Process of Storing and Accessing the Teaching Resources}

When the users access to data in the client, the information will be SSL-encrypted and sent to the Internet after the system safety authentication, then the sorter will fragment the data and transfer the pieces of data to the storage media in different physical locations scattered around using the cloud storage server management (Hong, Zhang \& Feng, 2011). When the users read or query data in the cloud storage server, the users can use the virtual view provided by the distributed storage management after authentication to complete data retrieval or delete, then 
the distributed storage management will operate on the corresponding equipments in the storage layer according to the record sheet, and finally return the operating results to the users.

The specific process of storing and accessing the teaching resources in the cloud environment is shown in Figure 6.
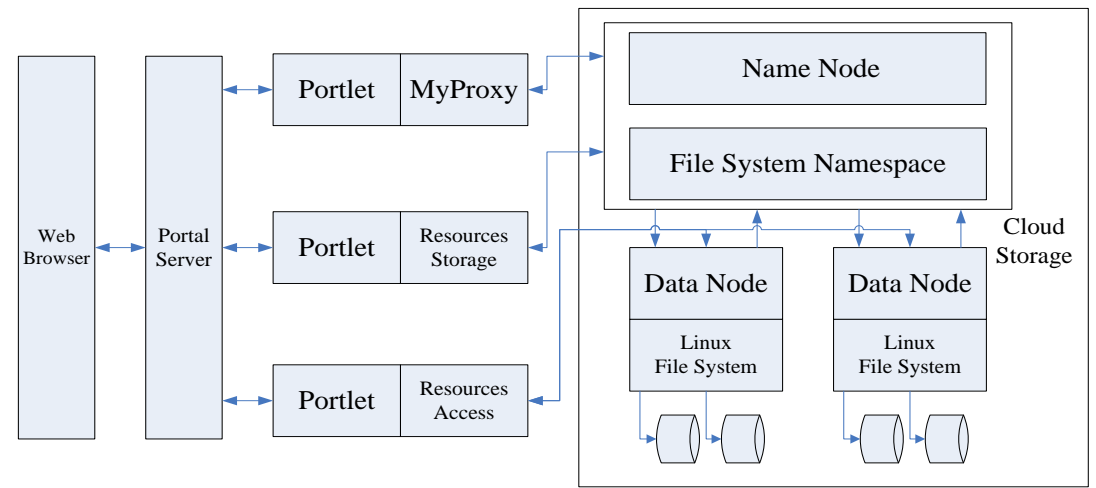

Figure 6. The process of storing and accessing the teaching resources in the cloud environment

- The users access the teaching resources page through a Web browser, and enter the user name and password first in the user authentication Portlet.

- The user's authentication is realized through MyProxy, and the users get the legal representation of the user's certificate from MyProxy according to the username and password provided by the users. This certificate represents the identity of the user, and then the users can legally access the appropriate application (Liu, Wang \& Wu, 2012).

- When accessing the resource storage services of Portlet, the users need to submit the path of local resources and the path of resource storage in the cloud.

- The storage service will cache these data in the temporary folder on the machine, and when the data block in the temporary folder reaches the Block value set (default is 64M), the Portlet will inform the name node in the cloud, then the name node will response the request of Portlet, and insert the file name of the resource into the file system hierarchy in the cloud and find a space for storing the data block in the data node, and tell the Portlet the information about the data node and the corresponding storage space. Then the Portlet will write the data block in the local temporary folder into the specified data node, and when the data block storage is finished and the data block in the temporary folder once again reaches the set of block value, the Portlet would apply to the storage space for the next data block. Due to the backup feature of Hadoop, each data block can have multiple copies stored in the other nodes in the cloud, and thus can improve the service scalability and fault tolerance. 


\subsection{Analysis of the Platform Performance}

The HDFS-based cloud storage is a dynamically adjustable and Internet-based storage solution, and the users can access storage targets using the network through a common and easy-to-use protocol and application programming interfaces. This new technology is useful for end users benefits. The cloud storage has the following advantages compared with the original teaching resources storage.

- Reliability. In the original way of teaching resources storage, if the resource corrupted for some reason, the users will not be able to access, so the reliability is not high. While the data storage in the cloud is distributed, and the teaching resources has been cut into multiple data blocks and scattered stored in the nodes of the cloud. Since there is a copy mechanism for the backup in the cloud storage, each piece of data has a number of backup storages on different nodes, so when a node in the cloud occurs an error, it will not affect the integrity of the resource because of the high reliability (Xu, Zhang \& Bi, 2012).

- Availability. The original teaching resources storage generally uses a single server, once the server encountered a problem, the users can neither store resources, but also unable to access resources. While the cloud storage has a distributed structure, the control node in the cloud continuously monitors the state of storage nodes through the heartbeat, and once finds that the storage node has failed, the control node will transfer the workload to those storage nodes running normally to complete. And this makes cloud storage with high availability (Agha, Kuhail, Abdelnabi, Abdelnabi \& Ghanim, 2011).

- The cost of storage. The original teaching resources storage generally uses professional storage devices, and due to the expensive price of the specialized storage devices, it also makes the cost of the resource storage very high. While most of the storage devices in the cloud are cheap business machines and they have greater storage capacity and lower storage costs compared to the specialized storage devices with a single large capacity.

- Scalability. We can easily change the storage capacity by dynamically adding and removing storage nodes in the cloud, while not affect the data. So the cloud storage has higher scalability than the original teaching resources storage.

- Automatic fault tolerance. Since the high loss rate of low-cost storage devices, the cloud storage can achieve automatic fault tolerance in the software layer instead of relying on the fault tolerance of the hardware itself.

- Management. It is helpful to the unified management of resources, and improves the resource utilization rate by storing the teaching resources in the cloud.

- Service Performance. In the original single-point centralized storage of the teaching resources, it is easy to cause the pressure of the equipment too large to affect the 
performance of the system when a large number of users store and access data at the same time. While the data in the cloud is stored distribute, so it can share the pressure of the storage and access, and thus improve the system performance (Liu, Sun, Wang \& Yang, 2012).

\section{Conclusions}

The development level and quality of education depend on the merits and efficiency in the use of teaching resources, especially in the case of obvious contradiction between the demand and supply of teaching resources. So to integrate teaching resources, improve the efficiency in the use of high quality teaching resources, and take the road of content development to enhance the competitiveness of education has become very important and urgent. As a new form of service, cloud storage can be a good solution to the integration of high quality teaching resources in universities. The HDFS-based cloud storage proposed in this paper is a dynamically adjustable and Internet-based storage solution, and the users can access storage targets using the network through a common and easy-to-use protocol and application programming interfaces. This new technology is useful for end users benefits. With the continuous development and improvement of cloud storage, it will necessarily result in more and more applications in the institutions of higher learning and education network.

\section{Acknowledgment}

Institute Level Projects Funded by Beijing Institute of Graphic Communication (E-b-2012-20).

\section{References}

Agha, S., Kuhail, I., Abdelnabi, N., Abdelnabi, M., \& Ghanim, A. (2011). Assessment of academic departments efficiency using data envelopment analysis. Journal of Industrial Engineering and Management, 4(2), 301-325. http://dx.doi.org/10.3926/jiem.2011.v4n2.p301-325

Bian, G., Gao, S., \& Shao, B. (2011). Security Structure of Cloud Storage Based on Dispersal. Journal of Xi'an Jiaotong University, 45(4), 41-45.

Chen, T. (2012). Application of HDFS cloud storage in digital resource integration of university. Electronic Design Engineering, 20, 4-6.

Fu, J., Chen, Z., \& Wang, J. (2012). Distributed Storage System Big Data Mining Based on HPC Application-A Solar Photovoltaic Forecasting System Practice. Information-An International Interdisciplinary Journal, 15(9), 3749-3755.

Gao, H., \& Wang, H. (2010). Research and Implementation of Educational Resources Integration based on Cloud. Modern Educational Technology, 20(3), 97-101. 
Hong, C., Zhang, M., \& Feng, D. (2011). Achieving efficient dynamic cryptographic access control in cloud storage. Journal on Communications, 32(7), 125-132.

Ji, H. (2003). Researching for Combination of the Resource of Science and Technology of Liaoning's College. Shenyang University of Technology, 2003.

Jia, Y. (2008). The Integration and Optimization of Teaching Resources in Beijing University of Chemical Technology. Beijing University of Chemical Technology.

Lin, H., \& Tzeng, W. (2012). A Secure Erasure Code-Based Cloud Storage System with Secure Data Forwarding. IEEE Transactions on Parallel and Distributed Systems, 35(3), 927-933.

Liu, J., Yu, D., \& Zhu, S. (2011). Research on new cloud storage service model. Application Research of Computers, 28(5), 1869-1872. http://dx.doi.org/10.1016/j.jnca.2011.03.010

Liu, Q., Wang, G., \& Wu, J. (2012). Secure and privacy preserving keyword searching for cloud storage services. Journal of network and computer applications, 35(3), 927-933.

Liu, Z., Sun, Q., Wang, S., \& Yang, F. (2012). The performance prediction of cloud service via JOGM $(1,1)$ model. AISS: Advances in Information Sciences and Service Sciences, 4(5), 70-77.

Lu, J., \& Shao, M. (2012). Trust Establishment for Data Integrity Checking in Cloud Computing. AISS: Advances in Information Sciences and Service Sciences, 4(14), 192-200.

Seppänen, M., \& Mäkinen, S. (2012). Resources in academic discourse: An empirical investigation of management journals. Journal of Industrial Engineering and Management, $3(1), 116-137$.

Wang, D. (2009). Research on the Integration of College Teaching Resources. Wuhan University of Technology.

Wu, J., Fu, J., Ping, L., \& Xie, Q. (2011). Study on the P2P Cloud Storage System. Acta Electronica Sinica, 39(5), 110-117.

Xu, L., Zhang, Y. \& Bi, X. (2012). A new model and queue management algorithm for congestion control in cloud service. AISS: Advances in Information Sciences and Service Sciences, 4(11), 320-327.

Journal of Industrial Engineering and Management, 2012 (www.jiem.org)

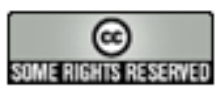

El artículo está con Reconocimiento-NoComercial 3.0 de Creative Commons. Puede copiarlo, distribuirlo y comunicarlo públicamente siempre que cite a su autor y a Intangible Capital. No lo utilice para fines comerciales. La licencia completa se puede consultar en http://creativecommons.org/licenses/by-nc/3.0/es/ 